\title{
Algebraic Set Theory and the Effective Topos
}

\author{
Claire Kouwenhoven-Gentil \\ and \\ Jaap van Oosten \\ Department of Mathematics, University of Utrecht \\ P.O.Box 80.010, 3508 TA Utrecht, The Netherlands \\ kouwenho@math.uu.nl jvoosten@math.uu.nl
}

September 2004, revised February 2005

\begin{abstract}
Following the book Algebraic Set Theory from André Joyal and Ieke Moerdijk [8], we give a characterization of the initial ZF-algebra, for Heyting pretoposes equipped with a class of small maps. Then, an application is considered (the effective topos) to show how to recover an already known model (McCarty $[9])$.
\end{abstract}

\section{Introduction}

When looking at models for unrestricted intuitionistic set theory IZF, one is naturally led to consider categorical models, since the internal logic governing categories is, in general, intuitionistic. In their book [8], André Joyal and Ieke Moerdijk proposed a new approach to set theory which is particularly suitable for categorical treatment, being essentially algebraic and entirely constructive.

They build a very general theory working for Heyting pretoposes with a natural number object, based on axioms for a "class of small maps". It turns out that, under some extra assumptions, the initial ZF-algebra for such a class of small maps is a model of IZF.

They invite the reader to explore in further details some examples of this theory and their "relation" with existing models in the literature. Our original aim was to investigate one of these examples: the effective topos $(\mathcal{E} f f)$. In the first section we focus on Heyting pretoposes equipped with such a class and prove a theorem characterizing the initial ZF-algebra among the ZF-algebras. The second section is devoted to an application of the theorem: we show that McCarty's realizability model of IZF can be embedded as an object of the effective topos and that it is isomorphic to the initial ZF-algebra model proposed by Joyal and Moerdijk. In other words, we make clear what was the nature of the relation between the models: they are in fact isomorphic.

Hence we see that an already known model of set theory is now given a framework to live in, giving us potentially new tools to investigate it as well as its underlying theory.

Acknowledgements. We would like to thank Benno van den Berg, Martin Hyland and Ieke Moerdijk for their interest in the topic and the useful discussions we had with them. 


\section{Characterization}

We follow the book by Joyal and Moerdijk [8] for the axioms for small maps, the definition of a ZF-algebra and the whole theory. Basic definitions are recalled in Appendix A.1. Hence, we will always consider a Heyting pretoposes with such a class of small maps (Other people have proposed alternative axiomatizations, most notably Awodey, Butz, Simpson and Streicher: see [1]).

We start by giving a characterization of the initial ZF-algebra.

Recall that given a ZF-algebra $(L, \sigma)$ a membership relation can be defined as follows: $y \epsilon x$ if and only if $\sigma(y) \leq x$. As usual, the notation $\mathcal{P}_{s}(L)$ is used to denote the object of "small subsets" of $L$.

Theorem 1.1 Let $\mathcal{E}$ be a Heyting pretopos and $S$ a class of small maps in $\mathcal{E}$. If $(L, \sigma)$ is a ZF-algebra satisfying:

1. (Extensionality) $\forall x, y(\forall z(z \epsilon x \rightarrow z \epsilon y) \rightarrow x \leq y)$

2. (Strict mono) $\forall x, y: \sigma(x) \leq \sigma(y) \rightarrow x=y$

3. (Smallness) $\forall x:\{y \in L \mid y \epsilon x\}$ is small, i.e. the map $\epsilon \longleftrightarrow L \times L \stackrel{\pi_{2}}{\longrightarrow} L$ is small

4. (Irreducibility of successors) $\forall x, \forall E \in P_{s}(L): \quad \sigma(x) \leq \bigvee_{y \in E} y \rightarrow \exists y \in E$ : $\sigma(x) \leq y$

5. ( $\epsilon$-induction) for any object $Z$ and any subobject $A$ of $Z \times L$, it holds in $\mathcal{E}$ : $\forall z \in Z[\forall x \in L(\forall y \epsilon x \quad((z, y) \in A) \rightarrow(z, x) \in A) \rightarrow \forall x \in L(z, x) \in A]$

then $L$ is the initial ZF-algebra.

Remark. The conditions 1.-5. are also necessary for $L$ to be initial: for conditions 2.-4. this is [8], Corollary II.1.4; for 1., [8], Proposition II.5.1 and 5. holds because if $L$ is initial in $\mathcal{E}$ then $Z \times L$ is initial in the slice $\mathcal{E} / Z$ for the class of small maps $S_{Z}$ defined in [8], section I.1 (this is implicit in [8], II.1.1 and the proof of mathematical induction II.5.1 can be adapted for $\epsilon$-induction).

Proof: We start by noting that conditions 1 . and 3 . imply that $\forall x \in L(x=$ $\left.\bigvee_{y \in x} \sigma(y)\right)$ holds.

Hence, for any homomorphism $\phi$ of ZF-algebras from $L$ to $(M, \tau), \forall x \in L(\phi(x)=$ $\bigvee_{y \epsilon x} \tau(\phi(x))$ holds. Conversely, if this last formula is true then $\phi$ is a homomorphism of ZF-algebras: since $\forall x \in L(\forall y \in L(y \epsilon \sigma(x) \leftrightarrow y=x))$ holds by 2. and the definition of $\epsilon$, also $\forall x \in L\left(\bigvee_{y \epsilon \sigma(x)} \tau(\phi(y))=\tau(\phi(x))\right)$ is true, which means that the map $\phi$ commutes with the successor operations. Commutation with small sups follows too, because for $E \in \mathcal{P}_{s}(L), \bigvee_{e \in E} \phi(e)=\bigvee_{e \in E} \bigvee_{y \in e} \tau(\phi(y))$ holds by assumption on $\phi$; moreover, the equalities

$$
\bigvee_{e \in E} \bigvee_{y \in e} \tau(\phi(y))=\bigvee_{y \in \bigvee E} \tau(\phi(y))=\phi(\bigvee E)
$$

hold by, respectively, assumption on $\phi, 4$. and assumption on $\phi$ again.

Now suppose that $\phi$ and $\psi$ are two homomorphisms of ZF-algebras from $L$ to $M$. Using 5. we can show that $\phi=\psi$ must hold: let $A=\{x \in L \mid \phi(x)=\psi(x)\}$ (and $Z=1$ ). From the fact that both $\phi$ and $\psi$ satisfy the identity just derived it is immediate that $A$ is inductive, so $A=L$ and $\phi=\psi$. 
So, there exists at most one homomorphism of ZF-algebras from $L$ to $M$. In order to prove that one exists, we first construct a 'transitive closure' operation in $L$. Let $T=\{x \in L \mid \forall y \epsilon x \forall z \epsilon y(z \epsilon x)\}$. Then define

$$
A=\{x \in L \mid \exists y \in L(y \in T \wedge x \leq y \wedge \forall z \in T(x \leq z \rightarrow y \leq z))\}
$$

We write $T C(x)$ for the unique $y \in L$, if it exists, which witnesses that $x \in A$ (note that $T C(x)$ is indeed uniquely determined). In order to see that $A$ is inductive, assume $\forall y \epsilon x(y \in A)$. By condition 3. and the uniqueness just mentioned we can form $x \vee \bigvee_{z \epsilon x} T C(z)$ and it is easy to see that this element witnesses that $x \in A$. So $A$ is inductive and we have a map $T C: L \rightarrow L$ with the expected properties.

In the following we shall often confuse an element $x \in L$ with the (small) subset $\{y \in L \mid y \epsilon x\}$; by 1. , this is legitimate.

Let $(M, \tau)$ be any ZF-algebra. Define the following subset of $L$ :

$$
B=\left\{x \in L \mid \exists ! f_{x}: T C(x) \rightarrow M \forall y \epsilon T C(x)\left(f_{x}(y)=\bigvee_{z \epsilon y} \tau\left(f_{x}(z)\right)\right)\right\}
$$

Note that the quantifier $\exists f_{x}$ is admissible: because $T C(x)$ is small and small maps are exponentiable, $M^{T C(x)}$ exists.

We prove that $B$ is inductive. Suppose $\forall y \epsilon x(y \in B)$. We have to find $f_{x}$ : $T C(x) \rightarrow M$. By construction, $T C(x)=x \vee \bigvee_{z \epsilon x} T C(z)$. Hence, by 4., $y \epsilon T C(x)$ is equivalent to $y \epsilon x \vee \exists z \epsilon x(y \epsilon T C(z))$.

For $y \epsilon x$ put $f_{x}(y)=\bigvee_{w \epsilon y} \tau\left(f_{y}(w)\right)$; for $y \epsilon T C(z)$, z $z x$ put $f_{x}(y)=f_{z}(y)$. This is well-defined, because if both cases apply $(y \epsilon x, y \epsilon T C(z), z \epsilon x)$ then by the induction hypothesis $y \in B$ and consequently the uniqueness of $f_{y}, f_{y}$ must agree with the restriction of $F_{z}$ to $T C(y)$, and hence

$$
\bigvee_{w \in y} \tau\left(f_{y}(w)\right)=\bigvee_{w \in y} \tau\left(f_{z}(w)\right)=f_{z}(y)
$$

A similar reasoning applies if for $z, z^{\prime} \epsilon x, y \epsilon T C(z)$ and $y \epsilon T C\left(z^{\prime}\right)$. Clearly, the map $f_{x}$ thus defined satisfies the condition in the definition of the set $B$, and it is unique with this property. So $x \in B$. We conclude that $B$ is inductive, so applying $5 ., B=L$.

We can now define a homomorphism of ZF-algebras $\phi: L \rightarrow M$ by $\phi(x)=$ $f_{\sigma(x)}(x)$. The verification that $\phi$ is a homomorphism of ZF-algebras is now straightforward, and left to the reader.

Remark 1.2 Note that as soon as Smallness holds, we can define two maps: Ext: $L \rightarrow P_{s} L$ and Int $: P_{s} L \rightarrow L$ which have the property that Extensionality + Strict mono + Irreducibility of successors hold if and only if (Ext,Int) forms an isomorphism. Ext $(x)$ is just defined by $\{y \in L \mid y \epsilon x\}$ and $\operatorname{Int}(E):=\bigvee_{y \in E} \sigma(y)$ (the notation is due to Moerdijk and Palmgren [11] and stands for respectively "externalization" and "internalization"). In other words, a ZF-algebra satisfying the smallness condition is initial if and only if it is a well-founded fixpoint w.r.t. $\epsilon$ and the maps Ext and Int (this is similar to other results on initial algebras in category theory: for example, suppose that $o: 1 \rightarrow X$ and $f: X \rightarrow X$ make the object $X$ into an algebra for the functor $(-)+1$. Then $X$ is with this structure a natural numbers object if and only if $\left[\begin{array}{l}f \\ o\end{array}\right]: X+1 \rightarrow X$ is an isomorphism and $X$ is well-founded w.r.t. the relation $\{(x, y) \mid y=f(x)\})$.

This characterization would not be very interesting if we would not have some applications in mind, but we do. In fact we will use the following corollary in the next section. 
Corollary 1.3 If in a topos we have an internal model $V$ of IZF and a class $S$ of IZF-small maps (i.e. a class of small maps satisfying three extra axioms: powerset, infinity and separation), then $V$ is the initial ZF-algebra if and only if it is $S$-complete (for the subset ordering, the set-theoretical union giving the supremum) and has the smallness- and the $\epsilon$-induction conditions. Note that the class $S_{V}$ of maps for which the supremum exists in $V$ along any map lacks only collection and representability to be a class of small maps (see [7], this is in fact true as soon as $V$ is a (sup) semi-lattice). In other words, $V$ is initial for $S$ if and only if $S$ is a subclass of $S_{V}$ containing $\epsilon \longleftrightarrow V \times V \stackrel{\pi_{2}}{\longrightarrow V}$ and $V$ satisfies the $\epsilon$-induction condition.

\section{Applications}

\subsection{The effective topos}

In Appendix A.2 we recall the definition of the effective topos $\mathcal{E} f f$ and some basic properties. Regarding the category Sets, we assume the existence of a strongly inaccessible cardinal $\kappa$ and the Axiom of Choice. This ensures that $\mathcal{E} f f$ has enough projectives, see [13].

As demonstrated in [8], section IV.4, the effective topos admits a class of IZFsmall maps and hence an internal model of IZF, $\mathcal{V}$. For more material on the effective topos, see [6], [12] and [13]. We will write $\Gamma \dashv \nabla$ for the geometric inclusion of Sets in $\mathcal{E} f f$ and we will use the following standard notations from recursion theory: "e.n $\simeq \phi_{e}(n) ", "\left\langle e_{0}, e_{1}\right\rangle=e "$ (see Appendix A.2 also for some notations from recusion theory).

\subsubsection{McCarty's model}

In his Ph.D. thesis [9], D.C. McCarty introduced a (non standard) realizability model of IZF, by giving an explicit definition of the underlying set and a transfinite inductive definition for truth in the model. Note that McCarty derived his model (as did Beeson in [2]) from earlier work by Friedman ([3]) and Grayson ([4]).

McCarty's model is defined as follows:

$$
\begin{aligned}
& V_{0}=\emptyset \\
& V_{\alpha+1}=P\left(\omega \times V_{\alpha}\right) \cong P \omega^{V_{\alpha}} \\
& V_{\lambda}=\bigcup_{\alpha<\lambda} V_{\alpha} \text { if } \lambda=\sup _{\alpha<\lambda} \alpha \\
& V_{\kappa}=\bigcup_{\alpha<\kappa} V_{\alpha}
\end{aligned}
$$

We say that $\mathcal{V}_{k} \models \phi$ iff there exists an $e$ such that $e \|-\phi$, where:

- $e \|-a \epsilon b$ iff there exists a $c$ such that $\left(\left\langle e_{0}, c\right\rangle \in b\right.$ and $\left.e_{1} \|-a=c\right)$

- $e \|-a=b$ iff for all $c, f:\left(\langle f, c\rangle \in a\right.$ implies that $e_{0} . f$ is defined and $e_{0} . f \|-c \epsilon b$ and $\langle f, c\rangle \in b$ implies that $e_{1} . f$ is defined and $\left.e_{1} . f \|-c \epsilon a\right)$

- $e \|-\phi \wedge \psi$ iff $e_{0} \|-\phi$ and $e_{1} \|-\psi$

- $e \|-\phi \vee \psi$ iff either $e_{0}=0$ and $e_{1} \|-\phi$ or $e_{0} \neq 0$ and $e_{1} \|-\psi$

- $e \|-\phi \rightarrow \psi$ iff for all $f:(f \|-\phi$ implies that e.f $\|-\psi)$

- $e \|-\neg \phi$ iff for all $f: \neg f \|-\phi$

- $e \|-\forall x \phi$ iff for all $a$ : $e \|-\phi(a)$ 
- $e \|-\exists x \phi$ iff there exists an $a$ such that $e \|-\phi(a)$

We will also write " $f \in a(c)$ " for $\langle f, c\rangle \in a$.

McCarty proved that:

Theorem 2.1 (McCarty) $\mathcal{V}_{\kappa}=I Z F$

\subsubsection{Embedding McCarty into $\mathcal{E} f f$}

In order to compare both models, we are going to embed McCarty's model into $\mathcal{E} f$. Define for each $\alpha \leq \kappa$ the following object of $\mathcal{E f f}$ :

$$
\mathcal{V}_{\alpha}:=\left(V_{\alpha},=\right)
$$

where, on the RHS, $[a=b]=\{e \mid e \|-a=b\}$. We need to check that, at every step, we get an object of $\mathcal{E} f f$ (i.e. that the definition of equality is, in terms of realizability, symmetric and transitive). We also want to make clear the relation between the external sets $V_{\alpha}$ and the corresponding internal $\mathcal{V}_{\alpha}$. The following proposition makes all of this clear (we obtain a uniform model, at each step being a quotient of the external construction):

Proposition 2.2 For all $\alpha \leq \kappa$ (hence in particular for $\kappa$ itself):

a) $\mathcal{V}_{\alpha}$ is uniform, $\mathcal{V}_{\alpha+1} \cong P\left(\mathcal{V}_{\alpha}\right)$, and

b) $=$ is an equivalence relation on $\nabla\left(V_{\alpha}\right)$, thus $\nabla\left(V_{\alpha}\right) \longrightarrow \mathcal{V}_{\alpha}$.

Proof: a) Using the recursion theorem, let $f$ be a solution of $f . n \simeq\langle n,\langle f, f\rangle\rangle$ and $e=\langle f, f\rangle$. In other words: $e=\langle\Lambda n .\langle n, e\rangle, \Lambda n .\langle n, e\rangle\rangle$.

From [12], we know that if $\mathcal{V}_{\alpha}$ is uniform, then $P\left(\mathcal{V}_{\alpha}\right)$ is also uniform, and isomorphic to $\left(P \omega^{V_{\alpha}},=\right)$, where $[f=g]=\bigcap\left\{[a=b] \rightarrow[f(a) \leftrightarrow g(b)] \mid a, b \in V_{\alpha}\right\}$ and where $[b \epsilon a]=\left[\exists b^{\prime} \in V_{\alpha}: a\left(b^{\prime}\right) \wedge b=b^{\prime}\right]$ on $\mathcal{V}_{\alpha} \times P\left(\mathcal{V}_{\alpha}\right)$. But this means exactly that $\mathcal{V}_{\alpha+1} \cong P\left(\mathcal{V}_{\alpha}\right)$. Moreover, if $e \in \bigcap_{b \in V_{\alpha}} E_{b}$ then given $a \in V_{\alpha+1}$ and $n \in a(b)$, then $\langle n, e\rangle \|-b \epsilon a$. In other words:

$\langle\Lambda n .\langle n, e\rangle, \Lambda n .\langle n, e\rangle\rangle \in E_{a}$. But this means: $e \in \bigcap_{a \in V_{\alpha+1}} E_{a}$.

Conclusion: $e \in \bigcap_{a \in V_{\kappa}} E_{a}$.

b) Reflexivity is realized by $\Lambda x . e$, and symmetry by $s=\Lambda x .\left\langle x_{1}, x_{0}\right\rangle$. For transitivity, we first consider the following:

$$
\begin{aligned}
& f(r, m, n, p)=\left\langle\left(m_{0} \cdot\left(\left(n_{0} \cdot p\right)_{0}\right)\right)_{0}, r \cdot\left\langle\left(n_{0} \cdot p\right)_{1},\left(m_{0} \cdot\left(n_{0} \cdot p\right)_{0}\right)_{1}\right\rangle\right\rangle \\
& g(r, s, m, n, p)=\left\langle\left(n_{1} \cdot\left(\left(m_{1} \cdot p\right)_{0}\right)\right)_{0}, s \cdot\left\langle r \cdot\left\langle s \cdot\left(\left(n_{1} \cdot\left(\left(m_{1} \cdot p\right)_{0}\right)\right)_{1}, s \cdot\left(\left(m_{1} \cdot p\right)_{1}\right)\right\rangle\right\rangle\right\rangle\right. \\
& H(r)=\Lambda n \Lambda m .\langle\Lambda p \cdot f(r, m, n, p), \Lambda p \cdot g(r, s, m, n, p)\rangle
\end{aligned}
$$

Let $r$ be a solution of $r . n \simeq H(r) . n$

We need to perform the following induction on $\alpha \leq \kappa$ :

$\forall a \in V_{\alpha}\left(\forall \beta \leq \kappa \forall \gamma \leq \kappa \forall b \in V_{\beta} \forall c \in V_{\gamma}: r \|-(a=b) \wedge(b=c) \rightarrow(a=c)\right)$

- $\alpha=0$ is trivial because $V_{0}=\emptyset$

- suppose it holds at rank $\alpha$, and $a_{1} \in V_{\alpha+1}, a_{2}, a_{3} \in V_{\kappa}$ and $\langle n, m\rangle \|-\left(a_{1}=\right.$ $\left.a_{2}\right) \wedge\left(a_{2}=a_{3}\right)$. Then, for $b, p$ such that $p \in a_{1}(b)$, we have:

$b \in V_{\alpha}$

$n_{0} \cdot p \|-b \epsilon a_{2}\left(=\exists c \cdot a_{2}(c) \wedge(b=c)\right)$

hence $m_{0} \cdot\left(\left(n_{0} \cdot p\right)_{0}\right) \|-c \epsilon a_{3}\left(=\exists d \cdot a_{3}(d) \wedge(c=d)\right)$

thus, by induction hypothesis for $b$ :

$\left\langle\left(m_{0} \cdot\left(\left(n_{0} \cdot p\right)_{0}\right)\right)_{0}, r \cdot\left\langle\left(n_{0} \cdot p\right)_{1},\left(m_{0} \cdot\left(\left(n_{0} \cdot p\right)_{0}\right)\right)_{1}\right\rangle\right\rangle \|-b \epsilon a_{3}$ 
In other words: $f(r, n, m, p) \|-b \epsilon a_{3}$.

On can show in almost the same way that for $b, p$ such that $p \in a_{3}(b)$, we have $g(r, s, m, n, p) \|-b \epsilon a_{1}$.

Conclusion: the property holds for $\alpha+1$.

- suppose the property holds $\forall \alpha<\lambda,\left(\lambda=\sup _{\alpha<\lambda} \alpha\right)$ and let $a \in V_{\lambda}$. Then there is a $\alpha_{a}<\lambda$ such that $a \in V_{\alpha_{a}}$ and the proposition holds already. Hence, this step is trivial.

Remark 2.3 In his thesis [9], McCarty already proved some properties, like the uniformity of the existence, in his model, by means of a "closure lemma" for $\epsilon$ and $=$. Unfortunately, we were not able to check this lemma, and, following the ideas in his thesis, we checked the proofs that it is a model of IZF again. Basically, when defining an element $f(a)$ in the model, depending on another element $a$ of the model, one has to check that it is well-defined, i.e. that $a=b \rightarrow f(a)=f(b)$ is valid.

\subsection{Two isomorphic models of IZF}

In this section we show that $\mathcal{V}_{\kappa}$ is in fact the initial ZF-algebra for the class of small maps in $\mathcal{E} f f$. Since it is already a model of IZF, the corollary of the preceding section tells us that it is enough to show that it is $S$-complete and satisfies both the smallness- and the $\epsilon$-induction conditions. However, for completeness, we start with some definitions in order to describe the ZF-algebra structure.

\section{Definition 2.4}

- "order" $\leq \longmapsto \mathcal{V}_{\kappa} \times \mathcal{V}_{\kappa}$, given by $L(a, b)=[\forall c(a(c) \rightarrow c \epsilon b)]$

- "successor" $\mathcal{V}_{k} \stackrel{\sigma}{\longrightarrow} \mathcal{V}_{k}$, given by $S(a, b)=[\forall c(c \epsilon b \leftrightarrow c=a)]$

- "supremum along small maps", given the following diagram:

$$
\begin{aligned}
(X, & =) \frac{f}{=[F]} \\
g & =[G] \\
(Y,=) &
\end{aligned}
$$

with $g$ small, we define the supremum of $f$ along $g$,

$$
\begin{aligned}
& (Y,=) \underset{=\left[M_{g}^{f}\right]}{\stackrel{\vee_{g} f}{\longrightarrow}} \mathcal{V}_{\kappa} \text { by } \\
& M_{g}^{f}(y, a)=\left[E_{y} \wedge \forall b(b \epsilon a \leftrightarrow \quad \exists x, c: G(x, y) \wedge F(x, c) \wedge b \epsilon c)\right] .
\end{aligned}
$$

We can now establish the following:

Proposition 2.5 $\left(\mathcal{V}_{k}, \leq, \bigvee, \sigma\right)$ satisifies the conditions of section 1.

Proof: (easy details on realizers are omitted)

- $\left(\mathcal{V}_{\kappa}, \leq, \bigvee, \sigma\right)$ is a ZF-algebra:

First, it is easy to see that $L$ defines a subobject of $\mathcal{V}_{\kappa} \times \mathcal{V}_{\kappa}$. Reflexivity for $\leq$ is realized by $\Lambda x . x_{0}$, antisymmetry by $\Lambda x . x$, and transitivity by $t$, where $t$ is a solution of $t .\langle x, y\rangle \simeq \Lambda p . f(r, x, y, p)$. Thus $\left(\mathcal{V}_{\kappa}, \leq\right)$ is a poset. 
Secondly, $S$ is a functional relation. For totality, we define $\sigma(a)(b)=[b=a]$ and show that $\bigcap_{a \in V_{\kappa}} S(a, \sigma(a)) \neq \emptyset$.

Third, $M_{g}^{f}$ is a functional relation. For totality, we define:

$a_{y}(b)=[\exists x, c: G(x, c) \wedge F(x, c) \wedge b \epsilon c]$ and show that:

$\bigcap_{y \in Y}\left[E_{y} \rightarrow M_{g}^{f}\left(y, a_{y}\right)\right] \neq \emptyset$. Here we have to show that there is indeed some ordinal $\beta$ lower than $\kappa$ sucht that $a_{y}$ can be defined in $V_{\beta}$. This needs some care: we will show in fact that $V$ is $S^{\prime}$-complete, where $S \subset S^{\prime}$ and $S^{\prime}=\{f \mid \Gamma f$ is small in Sets $\}$. As a consequence, $M_{g}^{f}$ defines a supremum.

- $\left(\mathcal{V}_{\kappa}\right.$ is $S^{\prime}$-complete) Let $f$ and $g$ be as follows, with $\Gamma g$ is small in Sets:

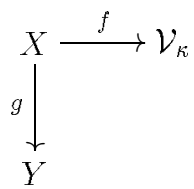

Moreover, we assume Sets $\models A C$ hence the quotient map $V_{k} \longrightarrow \Gamma\left(\mathcal{V}_{k}\right)$ has a section $s: \Gamma\left(\mathcal{V}_{\kappa}\right) \rightarrow V_{\kappa}$. Let $\bar{f}:=s \circ \Gamma f$. We obtain:<smiles>[In][Te]</smiles>

$\Gamma Y$

We will now define a map $h: \Gamma Y \rightarrow V_{\kappa}$. Its transpose $Y \longrightarrow \nabla V_{k}$ followed by the quotient map $\nabla V_{\kappa} \longrightarrow \mathcal{V}_{\kappa}$ in $\mathcal{E f f}$ will give us the supremum map $Y \rightarrow \mathcal{V}_{k}$. Let thus $[y]$ be in $\Gamma Y$. Since $\Gamma g$ is small, the set $A=\{[x] \mid(\Gamma g)[x]=$ $[y]\}$ has cardinality less than $\kappa$. In other words, there is a $\beta \leq \kappa$ such that for all $[x] \in A, \bar{f}[x] \in V_{\beta}$. The map we want can now be defined as:

$h[y]:=\left\{\langle n, z\rangle \mid \exists[x] \in(\Gamma g)^{-1}[y]: n \|-z \epsilon \bar{f}[x]\right\}$ as an element of $V_{\beta}$.

Hence $\left(\mathcal{V}_{\kappa}, \leq, \bigvee, \sigma\right)$ is a ZF-algebra.

- (smallness) it is easy to show that for all $\alpha<\kappa, V_{\alpha}$ is small in Sets. Hence for all $\alpha<\kappa, \mathcal{V}_{\alpha}$ is small in $\mathcal{E f f}$ (because $\nabla$ preserves small objects and using the quotient axiom). Then, if $a \in V_{\alpha+1}$, consider the following map in $\mathcal{E} f f$ :

$\{b \mid b \epsilon a\} \frac{f}{=[F]} \mathcal{V}_{\alpha}$ defined by: $F(b, c)=[b \epsilon a \wedge b=c]$

It is a well-defined (i.e. $F$ is a functorial relation) and it is clearly monic. We are done because the axiom of separation (every monic is small) holds in $\mathcal{E} f f$.

- $\epsilon$-induction: in [5], it is shown that $\Gamma$ preserves and reflects well-founded objects. Since $V_{\kappa}$ is built out of $\operatorname{Ord}_{\kappa}$ in Sets, the set of ordinals $\leq \kappa$, and $\leq$ is well-founded on $O r d_{\kappa}$, we can deduce $\epsilon$-induction.

Corollary 2.6 McCarty's model $\mathcal{V}_{k}$ is isomorphic to the model $\mathcal{V}$ proposed by Joyal and Moerdijk in $\mathcal{E} f$.

Remark 2.7 We worked with a classical metatheory (we used the character distinction of our ordinals in the construction and in the proofs). However, the results would still hold if we would start with a model of IZF (but we would not have anymore that, at every step, inside $\mathcal{E} f f, \mathcal{V}_{\alpha+1}=\mathcal{P}\left(\mathcal{V}_{\alpha}\right)$ ). Indeed, Grayson proved 
that defining ordinals as transitive sets of transitive sets (like Powell) still allow induction. It suffices then to define $V_{\alpha}=\bigcup_{\beta<\alpha} \mathcal{P}\left(\omega \times V_{\beta}\right)$. McCarty already noticed this fact, and that it can be extended for any pca $A$ (replacing $\omega$ by $|A|$ ). In other words: if $I Z F \vdash(A \models A P P)$ then $I Z F \vdash(\mathcal{V}(A) \models I Z F)$. But if we want to use the class of small maps given by Joyal and Moerdijk we have to assume Sets $\models A C$ and since $I Z F+A C=Z F$, this is not relevant for this paper. Hence the existence of the model is weaker that that of the class of small maps.

\section{Further research and open problems}

We are mainly interested in realizability. Some natural questions are: can we axiomatize IZF for realizability (as has been done for HA by Troelstra ([15]) and HAH by the second author ([12])? Can we develop some algebraic set theory for other realizability toposes (other than the ones coming from a pca, for which it works exactly the same way). So far we were not able to find a class of small maps for general toposes coming from triposes. For toposes arising as a glueing construction - note that in their articles dealing with algebraic set theory for CZF ([10] and [11]), Moerdijk and Palmgren hope that their axioms will be stable under glueing - we believe that Simpson's approach [14], with topos universes, is the correct one. As for realizability, recall that glueing Sets and $\mathcal{E} f f$ gives q-realizability and might gives us derived rules for IZF. More generally, we feel that some assumptions of weak choice principles might be needed to get representable classes of small maps (collection and representability pose problems). Besides, if we manage to understand how the internal logic of $\mathcal{E} f f$ relates to the logic of $\mathcal{V}$, as weak set theories (some results have already been found in this direction by Awodey, Butz, Streicher and Simpson, see [1]), we might also get results on choice principles for realizability. 


\section{A Appendix}

\section{A.1 small maps, ZF-algebras and models of IZF}

\section{small maps}

Let $\mathcal{E}$ be a Heyting pretopos with n.n.o and $\mathcal{S}$ a class of morphisms in $\mathcal{E}$. Consider the following axioms:

(S1) every isomorphism is in $\mathcal{S}$ and composition (if defined) of $\mathcal{S}$-maps gives a $\mathcal{S}$-map

(S2) every pullback of a $\mathcal{S}$-map along any other map is again a $\mathcal{S}$-map

(S3) (descent) Consider the following pullback:

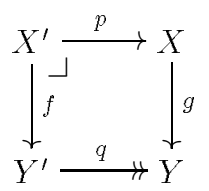

if $f \in \mathcal{S}$ and $q$ is epi then $g \in \mathcal{S}$

(S4) $0 \rightarrow 1$ and $1+1 \rightarrow 1$ are in $\mathcal{S}$

(S5) if $X \rightarrow Z$ and $Y \rightarrow Z$ are in $\mathcal{S}$ then so is $X+Y \rightarrow Z$

(S6) quotients Consider the following diagram:

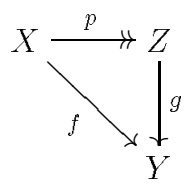

if $f \in \mathcal{S}$ and $p$ is epi then $g \in \mathcal{S}$.

(S7) (collection) if $X \stackrel{f}{\longrightarrow} Y \in \mathcal{S}$ and $Z \stackrel{g}{\longrightarrow} X$ is epi then there is a $\mathcal{S}$-map $P \stackrel{h}{\longrightarrow} Q$ such that the following diagram is a quasi-pullback:

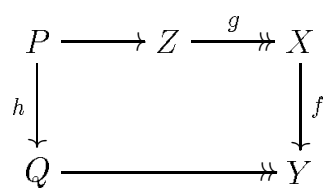

(S8) (exponentiability) every map is $\mathcal{S}$ is exponentiable

(S9) (representability) there is a map $\pi: E \rightarrow U$ which is universal in $\mathcal{S}$, that is, for every $\mathcal{S}$-map $f: X \rightarrow Y$ we have the following diagram, where both squares are pullbacks:

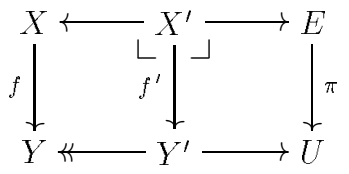




\section{Definition A.1}

- A class of maps satisfying axioms (S1) to (S8) is called a class of small maps

- An object of $\mathcal{E}$ is small if $X \rightarrow 1$ is a small map.

Proposition A.2 (Stability under slicing) Let $X$ be an object of a Heyting pretopos $\mathcal{E}$ equipped with a class $\mathcal{S}$ of small maps. Then the class $\mathcal{S}_{X}=\{f$ map of $\left.\mathcal{E} / X \mid \Sigma_{X}(f) \in \mathcal{S}\right\}$ is a class of small maps in $\mathcal{E} / X$. Besides, the functor $X^{*}$ preserves small maps and the universal small map.

Proposition A.3 (Representable small subobjects) For every object $X \in \mathcal{E}$, there is an object $P_{s}(X)$ representing the "families of small subobjects" of $X$, i.e. there is a $1-1$ correspondence between:

$$
\begin{aligned}
& S \longmapsto I \times X \stackrel{\pi_{1}}{\longrightarrow} I \text { small } \\
& \text { (a family of small subobjects of } X \text { with parameter } I \text { ), } \\
& \text { and } \\
& I \rightarrow P_{s}(X) \text {. }
\end{aligned}
$$

\section{IZF-small maps}

Consider now the following axioms:

(S'1) composition (if defined) of $\mathcal{S}$-maps gives a $\mathcal{S}$-map

(S9) (power-set) if $f: X \rightarrow Y \in \mathcal{S}$ then $P_{s}(f: X \rightarrow Y) \in \mathcal{S}_{Y}$, in $\mathcal{E} / Y$

(S10) (separation) every mono is in $\mathcal{S}$

(S11) (infinity) the natural number object is in $\mathcal{S}$.

\section{Definition A.4}

A class of maps satisfying axioms (S'1), (S2) to (S11) is called a class of IZF-small maps.

Proposition A.5 Both propositions above, stability under slicing and representable small subobjects, are still true for IZF-small maps.

\section{ZF-algebras}

Given a Heyting pretopos $\mathcal{E}$ with a class of small maps $\mathcal{S}$, we can define $\mathbf{Z F - a l g e b r a s}$ ("ZF" stands for Zermelo-Fraenkel):

Definition A.6 A ZF-algebra is an $\mathcal{S}$-complete sup-lattice $L$ in $\mathcal{E}$ together with a "successor" map $s: L \rightarrow L$. A homomorphism between two such algebras $(L, s)$ and $(M, t)$ is a map $f: L \rightarrow M$ commuting with successors and preserving supremas along small maps. We also define a membership relation on (generalized elements of) $L \times L$ by: $x \in y$ iff $s(x) \leq y$.

We write $(\mathcal{V}, s)$ for the free ZF-algebra (if it exists). We will also call it the "initial" ZF-algebra. Joyal and Moerdijk proved that the existence of a subobject classifier implies the existence of $\mathcal{V}$ (via bisimulations and well-founded forests, see chapter 3 of $[8])$. 


\section{A model of IZF}

First, let us recall the axioms of IZF as in [8]:

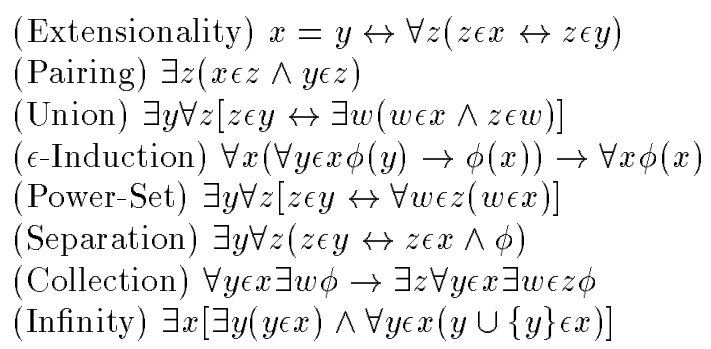

We can now recall the major result from [[8], p.65]:

Theorem A.7 Let $\mathcal{E}$ be a Heyting pretopos with a natural number object and $\mathcal{S}$ a class of IZF-small maps. Then the initial algebra $\mathcal{V}$ (if it exists) is a model of IZF.

\section{A.2 Realizability}

\section{the Effective topos: $\mathcal{E} f f$}

First, we recall some notations from recursion theory. $\langle.,$.$\rangle is a primitive recursive$ coding of pairs of natural numbers, with uncoding functions (.) o and (.) $)_{1}$. We will write partial recursive function application as . and use the $\Lambda$-notation: $\Lambda$ e.t means a standard code for the partial recursive function which gives $t$ at input $e$. Finally $e . n \simeq f . n$ means that $e$ and $f$ code the same partial function, that is, that the terms e.n and f.n are both defined or undefined, and if defined, then there are equal. Then, we introduce the effective topos:

Definition A.8 An object in the effective topos is a pair $(X,=)$, where $X$ is a set and $=$ a function from $X \times X$ into $P(\mathbb{N})$, that is, a relation on $X \times X$ for the realizability, which is symmetric and transitive (i.e., there exist realizers for the symmetry and the transitivity of $=$, or, in other words, symmetry and transitivity are valid).

An arrow $f:(X,=) \rightarrow(Y, \sim)$ in $\mathcal{E} f f$ is an equivalence class of functional relations, that is, $f=[F]$, where $F$ is a function from $X \times Y$ into $P(\mathbb{N})$, which is total, strict, relational and single-valued for the realizability. Two such $F$ and $G$ are equivalent iff $F(x, y) \leftrightarrow G(x, y)$ is valid.

There is a geometric inclusion of Sets into $\mathcal{E} f f$, generally written as $\Gamma \dashv \nabla$, where:

For a set $X, \nabla(X):=(X,=\nabla)$, where $\left[x=\nabla x^{\prime}\right]=\mathbb{N}$ if $x=x^{\prime}$, and $\left[x=\nabla x^{\prime}\right]=\emptyset$ otherwise.

For an object $(X,=)$ in $\mathcal{E} f f, \Gamma(X,=):=\{[x] \mid[x=x] \neq \emptyset\} / \sim$, where $[x] \sim\left[x^{\prime}\right]$ iff $\left[x=x^{\prime}\right] \neq \emptyset$.

Finally, we recall some particular objects:

- separated object/cover: an object $(X,=)$ is canonically separated if $[x=$ $\left.x^{\prime}\right] \neq \emptyset$ implies $x=x^{\prime}$ for all $x, x^{\prime} \in X$. For every object $\mathcal{X}=(X,=)$ in $\mathcal{E} f f$ we can define its canonically separated cover by $X_{s}=\left(X,=^{\prime}\right)$ where $\left[x={ }^{\prime} x\right]=[x=x]$ and $X_{s}$ is canonically separated. We write:

$$
X_{s} \longrightarrow \mathcal{X}
$$

$\mathcal{X}$ is separated iff $\mathcal{X}$ is isomorphic to a canonically separated object. 
- projective object/cover: an object $P$ is projective if given $P \rightarrow X$ and a cover $Y \longrightarrow X$, there is an arrow $P \rightarrow Y$ making the diagram commute. In $\mathcal{E} f$, we call $\mathcal{P}=(P,=)$ canonically projective if $\mathcal{P}$ is canonically separated and $\left[x=x^{\prime}\right]$ is a singleton for each $x \in P$. Then, projective objects are exactly those which are isomorphic to a canonically projective object. For every object $\mathcal{X}=(X,=)$ in $\mathcal{E} f f$ we can define its canonically projective cover by:

$X_{p}=\left(X \times \mathbb{N},=^{\prime}\right)$ where $\left[(x, n)=^{\prime}\left(x^{\prime}, n^{\prime}\right)\right]=\{n\}$ if $x=x^{\prime}$ and $n=n^{\prime}$ and $n \in[x=x]$, and is $\emptyset$ otherwise.

- uniform object: an object is uniform if it satisfies the uniformity principle as in [6]. We recall here results from [12], characterizing uniform objects:

Proposition A.9 The following are equivalent for an object $(X,=)$ of $\mathcal{E} f f$ :

(i) $(X,=)$ is uniform

(ii) $X$ is covered by a sheaf

(iii) For some $a, b \in \mathbb{N}$ :

$$
b \in \bigcap_{x \in X}\left[[x=x] \rightarrow \bigcup\left\{\left[x=x^{\prime}\right] \mid a \in\left[x^{\prime}=x^{\prime}\right]\right\}\right]
$$

(iv) $(X,=)$ is isomorphic to a $(Y,=)$ such that $\bigcap_{y \in Y}[y=y]$ is nonempty.

\section{Small maps in $\mathcal{E} f f$ and refinements}

In [8], Joyal and Moerdijk proved that the following class of maps satisfies all the axioms for IZF-small maps. We give now some refinements about this class, for further research, even if we will not really use them in this paper.

\section{Definition A.10}

- A map $f: X \rightarrow Y$ is ( $\kappa$-)small in $\mathcal{E} f f$ if there are projectives $P, Q$ such that the following diagram is a quasi-pullback, and $\Gamma(g)$ is ( $\kappa$-)small in Sets:

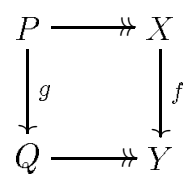

- An object $X$ is $(\kappa-)$ small in $\mathcal{E} f f$ if there is a projective cover $P$ of $X$ such that $\Gamma(P)$ is small in Sets.

Remark A.11 Given a small map $f: X \rightarrow Y$ and a projective cover $S$ of $Y$, we can find a projective $R$ to complete such a diagram as in the definition above. Indeed, take the pullback $Q \times_{Y} S$. Since $S$ is projective there is an arrow from $S$ to $Q \times{ }_{Y} S$. The pullback $R=S \times{ }_{Q} P$ satisfies the conditions of the diagram.

Proposition A.12 Let $K$ be a set of cardinality $\kappa$. Using the internal logic of $\mathcal{E} f$, a map $f: X \rightarrow Y$ is small iff $\forall y \in Y, \exists P \in P(\nabla K)$ such that:

1. there is an epi $P \rightarrow f^{-1}(y)$

2. there is no epi $\neg \neg P \rightarrow \nabla K$. 
Proposition A.13 A universal map can be given: $E \stackrel{\pi}{\longrightarrow} U, \pi$ being the obvious projection, by:

$U=\{(S, R) \mid S \in P(\nabla K), R$ equivalence relation on $S$ and

there is no epi $\neg \neg S \rightarrow \nabla K\}$

$E=\sum_{(S, R) \in U} S / R=\{(u, v) \mid u=(S, R) \in U, v \in S / R\}$.

\section{Proposition A.14}

(i) $f$ small in Sets iff $\nabla f$ small in $\mathcal{E} f f$

(ii) if $f$ small in $\mathcal{E} f f$ then $\Gamma f$ small in Sets

(iii) if $X$ separated then ( $X$ small in $\mathcal{E} f f$ iff $\Gamma X$ small in Sets)

(iv) $\forall X\left(X\right.$ small in $\mathcal{E} f f \Leftrightarrow \Gamma X$ small in Sets) iff $\forall X$ ( $\eta_{X}$ is small).

\section{References}

[1] S. Awodey, C. Butz, A.K. Simpson, and T. Streicher. Relating topos theory and set theory via categories of classes. Working draft, available at http://www.phil.cmu. edu/projects/ast/index .html, 2003.

[2] M.J. Beeson. Foundations of constructive mathematics. Springer-Verlag, 1985.

[3] H.M. Friedman. Some applications of Kleene's methods for intuitionistic systems. In A. R. D. Mathias and H. Rogers, editors, Cambridge Summer School in Mathematical Logic, pages 113-170. Springer-Verlag, 1973.

[4] R..J. Grayson. Heyting-valued models for intuitionistic set theory. In M. Fourman, C. Mulvey, and D.S. Scott, editors, Application of Sheaves, volume 743 of Lecture Notes in Mathematics, pages 402-414, Berlin, 1979. Springer.

[5] M. Hofmann, J. Van Oosten, and T. Streicher. Wellfoundedness in realizability. submitted; available at http: //ww . math.uu.nl/people/jvoosten/realizability/wellfreal .ps .gz, 2005 .

[6] J.M.E. Hyland. The effective topos. In A.S. Troelstra and D. Van Dalen, editors, The L.E.J. Brouwer Centenary Symposium, pages 165-216. North Holland Publishing Company, 1982.

[7] A. Joyal and I. Moerdijk. A categorical theory of cumulative hierarchies of sets. Comptes-Rendus Math., Soc. royale du Canada, 12:253-260, 1990.

[8] A. Joyal and I. Moerdijk. Algebraic Set Theory, volume 220 of London Mathematical Society Lecture Note Series. Cambridge University Press, Cambridge, 1995.

[9] D.C. McCarty. Realizability and recursive mathematics. Technical Report CMU-CS-84-131, Department of Computer Science, Carnegie-Mellon University, 1984. Report version of the author's PhD thesis, Oxford University 1983.

[10] Teke Moerdijk and Erik Palmgren. Wellfounded trees in categories. Annals of Pure and Applied Logic, 104:189-218, 2000.

[11] Ieke Moerdijk and Erik Palmgren. Type theories, toposes and constructive set theory: predicative aspects of AST. Annals of Pure and Applied Logic, 114:155-201, 2002. 
[12] Jaap van Oosten. Axiomatizing higher-order Kleene realizability. Annals of Pure and Applied Logic, 70:87-111, 1994.

[13] E.P. Robinson and G. Rosolini. Colimit completions and the effective topos. Journal of Symbolic Logic, 55:678-699, 1990.

[14] A.K. Simpson. Elementary axioms for categories of classes. In Proc. 14th Annual IEEE Symposium on Logic in Computer Science, pages 77-85, 1999.

[15] A.S. Troelstra, editor. Metamathematical Investigation of Intuitionistic Arithmetic and Analysis. Springer (Lecture Notes in Mathematics 344), 1973. With contributions by A.S. Troelstra, C.A. Smoryński, J.I. Zucker and W.A. Howard. 